\title{
Phenomenology of $K^{-}$atoms and other strange hadronic atoms
}

\author{
A. Gal ${ }^{a}$ \\ ${ }^{a}$ Racah Institute of Physics, The Hebrew University, Jerusalem 91904, Israel
}

\begin{abstract}
Recent optical-potential studies of the phenomenology of $K^{-}$atoms are reviewed. It is shown that the data can be fitted by a complex optical potential with either a relatively shallow attractive component $(\sim-50 \mathrm{MeV}$ at $\left.\rho_{0}\right)$, as suggested by a self consistent application of chirally motivated coupled-channels models, or a relatively deep attractive component $(\sim-180$ $\mathrm{MeV}$ at $\rho_{0}$ ) as suggested by a mean-field extrapolation of a phenomenological low-density expansion. Both classes of these optical potentials, due to their strongly absorptive component, predict similarly structured relatively narrow 'deeply bound' $K^{-}$atomic states with widths saturating at about $2 \mathrm{MeV}$. Possible formation reactions are briefly discussed. The more speculative case for relatively narrow deeply bound $K^{-}$nuclear states is briefly mentioned. Recent works on other strange hadronic atom systems, for the $\Sigma^{-}$and $\Xi^{-}$hyperons, are also discussed.
\end{abstract}

Invited talk presented at HYP2000 in Torino, October 2000. To appear in Nuclear Physics A.

November 5, 2018 


\section{KAONIC ATOMS}

In this section I review recent works on the $K^{-}$nucleus optical potential, with emphasis in the first two subsections on fitting it to the 'normal' X-ray data in kaonic atoms, and in the third subsection on predictions of narrow 'deeply bound' $K^{-}$atomic states.

\section{A. Hybrid relativistic mean field approach to $K^{-}$atoms}

Here I report on the recent work by the Jerusalem-Řež collaboration [1]. Antikaons are incorporated into this relativistic mean field (RMF) model by using the Lagrangian density of the form [2]

$$
\mathcal{L}_{K}=\partial_{\mu} \bar{\psi} \partial^{\mu} \psi-m_{K}^{2} \bar{\psi} \psi-g_{\sigma K} m_{K} \bar{\psi} \psi \sigma-i g_{\omega K}\left(\bar{\psi} \partial_{\mu} \psi \omega^{\mu}-\psi \partial_{\mu} \bar{\psi} \omega^{\mu}\right)+\left(g_{\omega K} \omega_{\mu}\right)^{2} \bar{\psi} \psi,
$$

describing the interaction of the antikaon field $(\bar{\psi})$ with the scalar $(\sigma)$ and vector $(\omega)$ isoscalar fields. The corresponding equation of motion for $K^{-}$in a $Z=N$ nucleus can be expressed by a Klein-Gordon equation with the real part of the optical potential given at threshold by

$$
\operatorname{Re} V_{\mathrm{opt}}=\frac{m_{K}}{\mu}\left(\frac{1}{2} S-V-\frac{V^{2}}{2 m_{K}}\right),
$$

where $S=g_{\sigma K} \sigma(r)$ and $V=g_{\omega K} \omega_{0}(r)$ in terms of the mean isoscalar fields, and where $\mu$ is the $K^{-}$nucleus reduced mass. Note that for antikaons, the vector potential $V$ contributes attraction, just opposite to its role for kaons and nucleons. Each of the three terms on the r.h.s. of Eq. (2), thus, gives rise to attraction. Consequently, it becomes impossible to satisfy the low density limit which requires that, due to the subthreshold $\Lambda(1405)$, Re $V_{\text {opt }}>0$ as $\rho \rightarrow 0$. For nuclei with $N>Z$, the potential should include also an isovector part due to the interaction of the $K^{-}$with the $\rho$ meson field. However, this was omitted from the present calculations as it was found in previous analyses of kaonic atoms [3 5] to have a marginal effect. Furthermore, since the imaginary part of $V_{\text {opt }}$ is not directly addressed in the RMF approach, a phenomenological $t \rho$ parameterization was used for it, after making sure that a more involved density dependence of Im $V_{\text {opt }}$ does not introduce further effects.

In order to construct the RMF Re $V_{\text {opt }}$ of Eq. (2), one needs to specify $\alpha_{\sigma}$ and $\alpha_{\omega}$, where $\alpha_{m}=g_{m K} / g_{m N}$. Treating both $\alpha_{\sigma}$ and $\alpha_{\omega}$ as free parameters, the best fit potential consists of a strongly attractive vector potential and a strongly repulsive scalar potential, quite far from the structure expected e.g. from the naive quark model (QM) [6]. The corresponding Re $V_{\text {opt }}$ provides a very good fit to the atomic data. It is attractive in the nuclear interior, reaching a depth of about $190 \mathrm{MeV}$ in close agreement with the phenomenological densitydependent (DD) potentials of Ref. [5], and it becomes repulsive at large distances, thus reflecting a posteriori effects of the $\Lambda(1405)$ at low densities.

The RMF description is well justified within the nuclear interior, for densities larger than about $0.2 \rho_{0}$ where the effects of the $\Lambda(1405)$ may be neglected, as demonstrated in Refs. [7 9]. The existence of the $\Lambda(1405)$ resonance clearly poses a difficulty for the RMF approach at low densities if the parameters $\alpha_{m}$ are allowed to deviate only moderately from the values suggested by the underlying hadron symmetries. In the hybrid model of Ref. [1], the functional RMF form Eq. (2) was used in the nuclear interior for $\operatorname{Re} V_{\text {opt }}$, whereas a 
purely phenomenological DD form [3,4] was used in the surface of the nucleus and beyond. The sensitivity to the choice of the radius $R_{M}$, where the two forms are matched to each other, was checked and found to be small. The density $\rho\left(R_{M}\right)$ is sufficiently high to justify using the RMF approach, and sufficiently low so that the atomic data are still sensitive to the RMF form. Figure 4 of Ref. [5], and in particular Fig. 3 of Ref. [10] for Ni, show that by analyzing kaonic atoms, using a given functional form for $V_{\mathrm{opt}}$, one determines the real part of the $K^{-}$nucleus DD optical potential up to $\rho=0.9 \rho_{0}$. This is well above the density at which the RMF form takes over in the present approach.

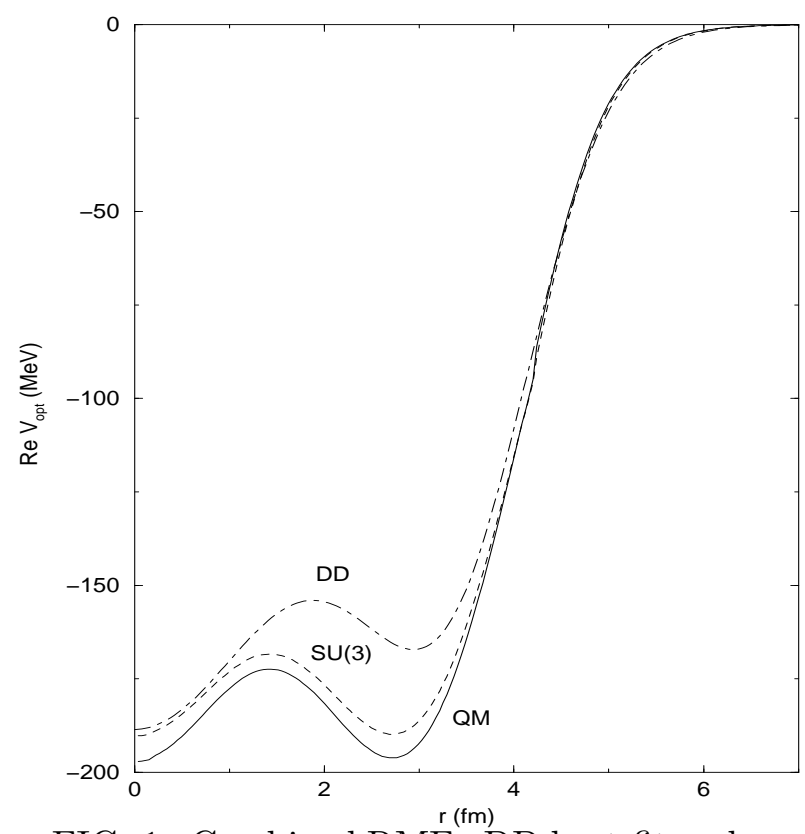

FIG. 1. Combined RMF+DD best-fit real potentials for $K^{-}$in $\mathrm{Ni}$, using the QM and $\mathrm{SU}(3)$ versions. Also shown is the phenomenological DD potential.

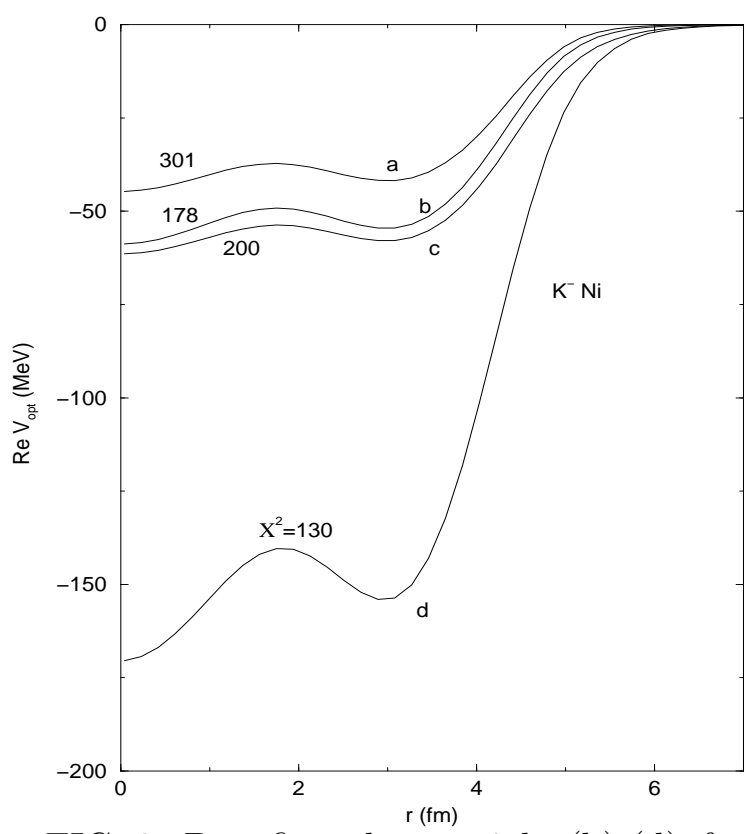

FIG. 2. Best-fit real potentials, (b)-(d), for $K^{-}$in $\mathrm{Ni}$ using the self consistent potential (a) due to Ramos and Oset. The values of total $\chi^{2}$ are given for each curve.

Fitting to the $K^{-}$atomic data, the vector coupling constant ratio $\alpha_{\omega}$ was kept fixed, guided by theoretical considerations, and the scalar coupling constant ratio $\alpha_{\sigma}$ was varied together with the parameters of the DD real potential form (for $r>R_{M}$ ). For the coupling constant $g_{\omega K}$, either the constituent QM value $\alpha_{\omega}=\frac{1}{3}$ was used, or the $\mathrm{SU}(3)$ relation $2 g_{\omega K}=g_{\rho \pi}=6.04$. The resulting scalar potentials are attractive, of the order of magnitude expected (see Table I in Ref. [1]). These best-fit hybrid RMF+DD potentials describe the data very well, with $\chi^{2} / N$ values of $1.4-1.5$.

Figure [1 shows the hybrid RMF+DD best fit real potentials of Ref. [1] for $\mathrm{Ni}$, using the $\mathrm{QM}$ and $\mathrm{SU}(3)$ options. It is seen that the depths of the hybrid real potentials in the nuclear interior for the different choices of $\alpha_{\omega}$ are about $180 \mathrm{MeV}$, very close to the depth of the purely phenomenological DD potential, also shown in the figure. These depths are to be compared to those displayed in Fig. 2 discussed below. We note that omitting the $V^{2}$ term in Eq. (2), and refitting the $K^{-}$atomic data, the resulting depths are smaller than the RMF+DD depths shown in Fig. 1 by less than $10 \mathrm{MeV}$. Finally, as for Im $V_{\text {opt }}$, it is well determined in all of the above best fits, with a depth of about $60 \mathrm{MeV}$, not far from the value expected from the low density limit. 


\section{B. Self consistent optical potentials in chirally inspired models}

In this approach (reviewed by Ramos in these proceedings) chirally motivated coupledchannel $t$ matrices in the $S=-1$ strangeness sector, including the $\bar{K} N$ channels, were fitted to the near-threshold data and used to construct a $\tilde{t} \rho K^{-}$nucleus optical potential [8,9]. Here $\tilde{t}$ is a medium-modified $\bar{K} N t$ matrix incorporating Pauli blocking in the intermediate nucleon states, nucleon and hyperon dispersive corrections and also, recently, self consistency 111,12 (and [13 within a non chiral model). This latter requirement of self consistency means that the output $V_{\text {opt }}$ is used in the $\bar{K}$ nuclear-medium propagator within the Lippmann Schwinger equation to generate the $\tilde{t}$ matrix, and therefore also the input $V_{\text {opt }}$, from the coupled-channel interaction $v$. A common feature of the self consistent evaluations is that Re $V_{\text {opt }}$ is rather shallow, typically about $-40 \mathrm{MeV}$ at $\rho_{0}$, as shown by curve $(a)$ in Fig. 2.

The $K^{-}$nucleus optical potential derived self consistently by Ramos and Oset [12 has been applied in Ref. [14 to a subset of the $K^{-}$atomic data (including the same $2 p, 3 d$ and $4 f$ sequences already covered in Ref. [15]) which it reproduces semi-quantitatively. Baca et al. [16] have recently extended this analysis, including the missing sequences for heavier atoms, and also allowing for an additional phenomenological component of a $(\delta t) \rho$ form. Figure 2, taken from a work in progress [17], shows the successively decreasing total $\chi^{2}$ values for $N=65$ data points, plus the calculated depths of Re $V_{\text {opt }}$, when such addition is allowed (case $b$ ). The potential has become somewhat more attractive, reaching about $-60 \mathrm{MeV}$ at $\rho_{0}$. Shown also are two more searches, starting with the Ramos and Oset $V_{\text {opt }}$ (case $\left.a\right)$. In the first case $(c)$, the $I=0$ component which is intimately related to the $\Lambda(1405)$ propagation and dissolution in the medium is kept as is, whereas the strength of the $I=1$ component which is not constrained well by the low-energy $\bar{K} N$ data is allowed to get renormalized, while keeping its 'theoretical' shape intact. This kind of a search is somewhat less successful than searching on a $(\delta t) \rho$ form, yielding a similar depth for Re $V_{\text {opt }}$. Trying a combination of the above two modifications (case $d$ ) reduces the value of $\chi^{2}$ considerably, close to that due to the completely phenomenological DD analysis [5], producing a very deep potential (about $-170 \mathrm{MeV}$ at $\rho_{0}$ ). Similar results are obtained if one starts from the self consistent $K^{-}$nucleus potential of Ref. [13].

\section{Narrow deeply bound $K^{-}$and $\bar{p}$ atomic states}

Friedman and Soff [18] and Toki and Yamazaki [19] predicted that $\pi^{-}$atomic levels remain relatively narrow and well resolved, with absorption widths smaller than $1 \mathrm{MeV}$, down to the $1 s$ level in $\mathrm{Pb}$. This narrowness is due to the repulsive $s$-wave pion interaction which 'pushes' its wave function outside of the nucleus, thus suppressing the absorption width. Recently the $1 s$ and $2 p \pi^{-}$'deeply bound' atomic states (DBAS), which are inaccessible via the atomic cascade process, have been observed at GSI [20] using the recoilless $\left(d,{ }^{3} \mathrm{He}\right)$ reaction on two $\mathrm{Pb}$ isotopes, confirming the theoretical prediction.

For $K^{-}$and $\bar{p}$ atoms, Re $V_{\text {opt }}$ is strongly attractive and, furthermore, Im $V_{\text {opt }}$ is particularly strong (50 - $100 \mathrm{MeV}$ deep [5]), so DBAS a-priori are unlikely to be narrow. However, as shown recently, a strongly absorptive potential makes $V_{\text {opt }}$ effectively repulsive, and the atomic wave functions are then substantially suppressed within the nucleus, with the atomic 
level widths saturating as function of Im $V_{\text {opt }}$. Figures 3-6 below demonstrate these points 21,22, 2 .

Figure 3 shows calculated energy levels for kaonic $\mathrm{Pb}$. The bars stand for the full width $\Gamma$ of the level. The $7 i \mathrm{~K}^{-}$level in $\mathrm{Pb}$ is the last observed in the X-ray spectrum. Clearly the deeper levels are quite well defined even for a heavy nucleus, but $l$-selective reactions are needed to observe these DBAS. These predictions are insensitive to details of $V_{\text {opt }}$, provided it was fitted to the 'normal' levels derived from X-ray spectra.

Figure 4 shows the moduli squared of $2 p$ radial wave functions in kaonic $\mathrm{Pb}$. The dashed curve (C) is for the finite size Coulomb potential only. The solid curve $(\mathrm{F})$ is for the full $V_{\text {opt }}$ added and it shows essentially total expulsion of the wave function from the nucleus, whose r.m.s. radius is $5.5 \mathrm{fm}$, thus reducing dramatically the width of the level. The dotted curve $(\mathrm{Im})$ is when only $\mathrm{Im} V_{\text {opt }}$ is added, showing that it is dominant in determining the wave function. The dot-dashed curve (Re) shows the wave function when only the strongly attractive Re $V_{\text {opt }}$ is added. The three small inner peaks preceding the main peak well outside the nucleus indicate that three nuclear bound states exist in this real potential, causing the atomic wave function (by orthogonality) to develop nodes. This inner structure then causes the main atomic peak of the wave function to shift to larger radii compared to the Coulomb wave function, thus resulting in a repulsive shift. The node structure inside the nucleus depends critically on Re $V_{\text {opt }}$ and so are the energies of the broad nuclear bound states. The atomic part of the wave function is much less sensitive.

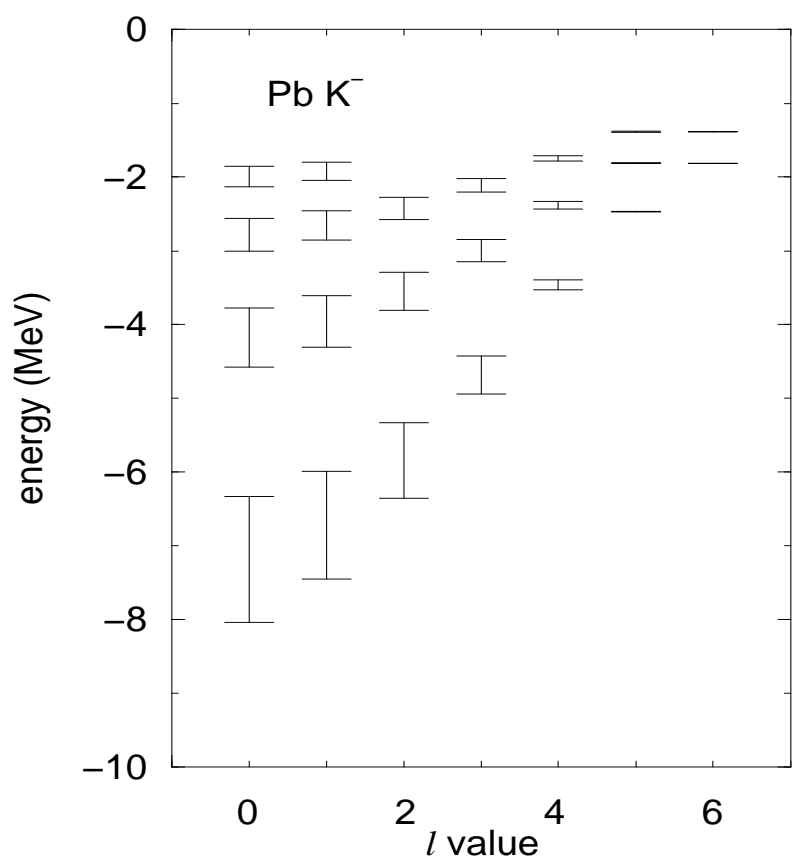

FIG. 3. Energy levels for $K^{-}$atomic $\mathrm{Pb}$.

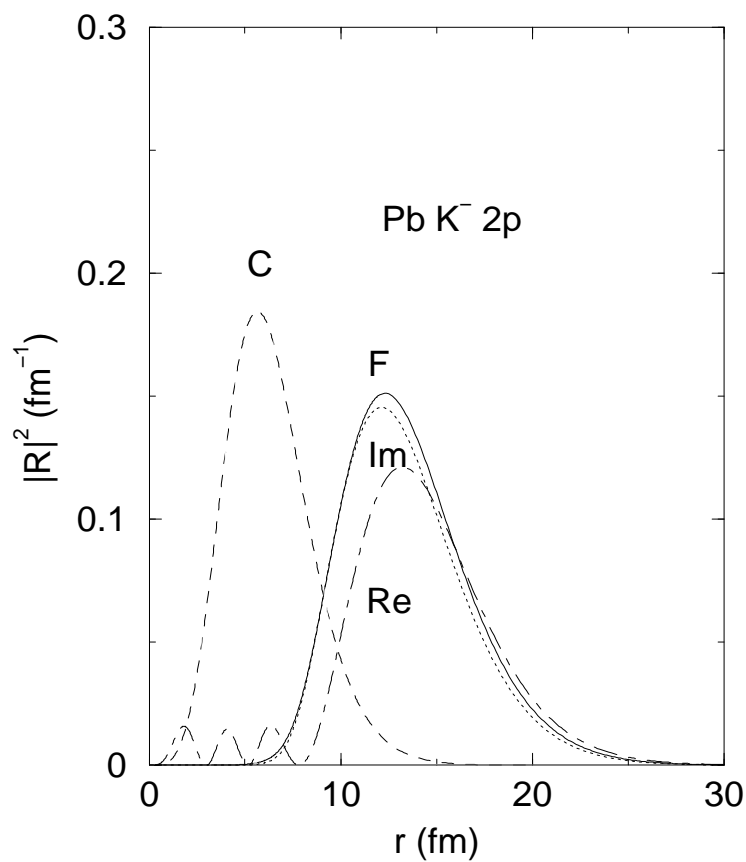

FIG. 4. Squared $2 p$ radial wavefunctions in $K^{-}$atomic $\mathrm{Pb}$.

The saturation of atomic level widths may be demonstrated by studying the dependence of strong interaction level shifts $\epsilon$ and widths $\Gamma$ on $\operatorname{Im} V_{\text {opt }}$. Figure 5 shows calculated shifts and widths for the $2 p$ level in kaonic $\mathrm{Pb}$ as function of $\operatorname{Im} b_{0}$ when $\operatorname{Re} b_{0}$ is being held at its nominal value, using a $t \rho$ potential with effective scattering length $b_{0}$. It is seen that at about $20 \%$ of the nominal value of $\operatorname{Im} b_{0}$ (of $0.92 \mathrm{fm}$ ) the width already saturates and then 
it goes slowly down, while the shift stays essentially constant at a very large repulsive value, in spite of the strongly attractive $\operatorname{Re} V_{\text {opt }}$. The mechanism behind the saturation of widths is fairly independent of $\operatorname{Re} V_{\mathrm{opt}}$, as demonstrated in the previous figure. In the absence of data on this part of the atomic spectrum, the validity of the saturation property is supported by studying total reaction cross sections at very low energies. Related data do exist for $\bar{p}$ annihilation on a few nuclear targets. Figure 6 shows calculated total reaction cross sections for $\bar{p}$ at $57 \mathrm{MeV} / \mathrm{c}$ on ${ }^{4} \mathrm{He}$ and $\mathrm{Ne}$ as function of $\operatorname{Im} b_{0}$ [23,24]. The measured cross sections (not shown in the figure) are well reproduced by the plateau values. The striking similarity between Fig. 5 and Fig. $[$ is due to the observation that both $\Gamma$ at negative energies, and $\sigma_{R}$ at positive energies, involve averaging $\operatorname{Im} V_{\text {opt }}(r)$ with $|\psi|^{2}$. Therefore, in the strongabsorption limit, when $|\psi|^{2}$ is pushed out of the nuclear domain (as shown in Fig. 国) these observables cease to rise approximately linearly with the strength of $\operatorname{Im} V_{\mathrm{opt}}$.

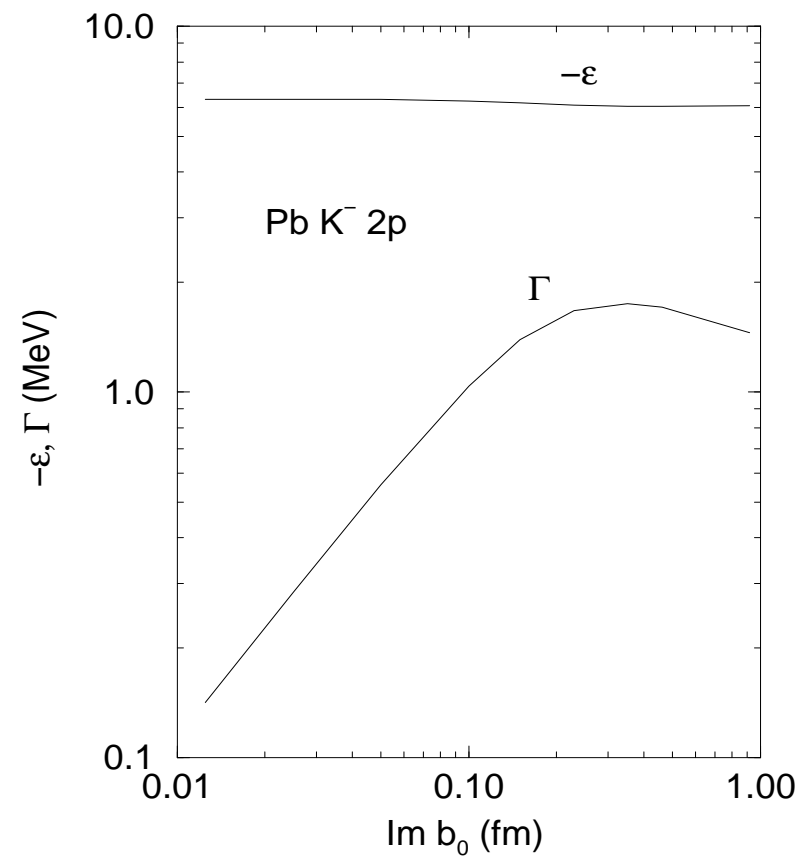

FIG. 5. Strong interaction shifts and widths

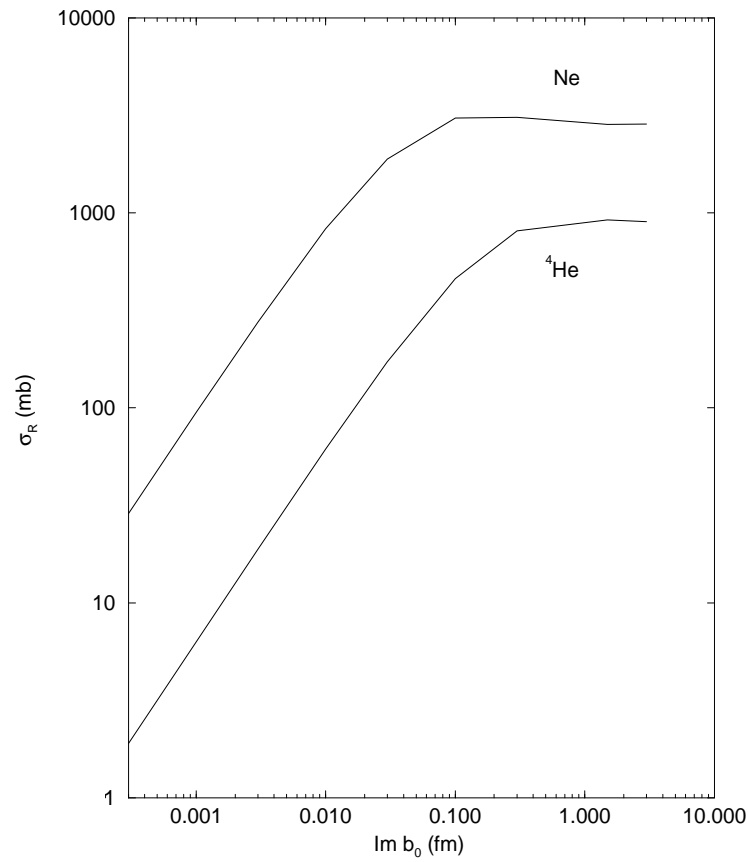

FIG. 6. Total reaction cross sections for 57

as function of $\operatorname{Im} b_{0}$ for the $2 p$ level in kaonic $\mathrm{MeV} / \mathrm{c} \bar{p}$ on ${ }^{4} \mathrm{He}$ and Ne as function of $\operatorname{Im} b_{0}$. $\mathrm{Pb}$. Re $b_{0}=0.62 \mathrm{fm}$.

The lesson gained from discovering $\pi^{-}$DBAS [20] is that nuclear reactions with momentum transfer $q$ below $50 \mathrm{MeV} / \mathrm{c}$ should suit best the production of $K^{-}$DBAS. First, the suppressive effects of the nuclear absorption on low- $q$ production cross section are weaker than for reactions with higher values of $q$ [25] and second, the angular momentum transfer is then minimal: $\Delta l \sim 0$, thus ensuring selectivity.

Low energy $(16 \mathrm{MeV}) K^{-}$mesons are now available at DA $\Phi$ NE due to the decay at rest $\phi(1020) \rightarrow K^{+} K^{-}$. This corresponds to $q\left(0^{\circ}\right)=47 \mathrm{MeV} / \mathrm{c}$ in the $\left(K^{-}, p\right)$ reaction. Although forward cross sections as large as $0.1 \mu \mathrm{b} / \mathrm{sr}$ may be expected, a major problem would be the need to detect unambiguously the low energy outgoing proton. Somewhat smaller cross sections are expected for the $\left(K^{-}, \gamma\right)$ reaction [14] in which $q\left(0^{\circ}\right)$ is about twice as large. Here too, the expected background at these low energies poses a serious problem. If the decay at rest of the $\phi(1020)$ meson were to take place at close proximity to 
a nucleus such that the kaonic atom recoiled as a whole, then the signature would be a peak in the $K^{+}$energy spectrum above the $32 \mathrm{MeV}$ released in the free-space $\phi$ decay. However, since $q \sim 180 \mathrm{MeV} / \mathrm{c}$ in this at-rest $\left(\phi, K^{+}\right)$reaction, it is not a favorable situation. It would be interesting to look for secondary nuclear interactions of the $\phi$ meson in flight prior to its decay, e.g. the recoilless $\left(\phi, K^{+}\right)$reaction which attaches a $K^{-}$meson to the nuclear target [22].

Several authors [26,27] have drawn attention to $K^{-}$nuclear states bound by a very deep potential, similar to the best-fit DD potential of Batty et al. [5]. The deepest states are then blocked from decaying by the two-body mode $\bar{K} N \rightarrow \pi \Sigma$ and their width could then be reduced to $10 \mathrm{MeV}$. However, about $20 \%$ of $K^{-}$absorption at rest is due to nonpionic $K^{-} N N$ modes, releasing substantially larger energy than the corresponding binding energies. A rough estimate for the residual width, largely due to these modes, is $30-40 \mathrm{MeV}$. It is unlikely then that well separated signals could exist in the outgoing nucleon spectrum of the $\left(K^{-}, N\right)$ reaction, except perhaps in the very special case of kaonic He atoms [27]. Considering the production cross sections anticipated for such deeply bound $K^{-}$nuclear states, we disagree with the estimate made by Kishimoto [26] for the $\left(K^{-}, p\right)$ reaction at $1 \mathrm{GeV} / \mathrm{c}$. Our estimate is at the few $\mu \mathrm{b} / \mathrm{sr}$ level only. It would be extremely difficult to observe these relatively broad states given such low integrated cross sections.

\section{II. $\Sigma^{-}$ATOMS}

DD analyses of $\Sigma^{-}$atoms [28,29] were already reviewed in the HYP97 meeting [10]. These analyses suggested for the first time that the $\Sigma$ nucleus interaction, which is attractive outside the nucleus, is likely to be repulsive within. Dabrowski subsequently suggested that such repulsion indeed is required by the shape of $\Sigma$ quasi-free excitation spectra [30] (see also his contribution in these proceedings). Another element that emerged clearly from these works was that the isovector, Lane potential component of the $\Sigma$ nucleus optical potential was substantial and of a definite sign. In Ref. [29], this derivation was assigned to the particularly accurate shift and width values measured for the atomic $9 k \Sigma^{-}$level in $\mathrm{Pb}$. Very recently, Loiseau and Wycech [31] showed that the relatively accurate $10 l$ small width value, derived from the yield measurement, is instrumental in extracting the value of the $\pi \Lambda \Sigma$ coupling constant, assuming that the $\Sigma^{-}$absorption occurs peripherally on protons, $\Sigma^{-} p \rightarrow \Lambda n$, via pion exchange. The value thus deduced, incorporating several other upperlevel widths (of order eV) which are quite justifiedly evaluated perturbatively, is

$$
\frac{f_{\pi \Lambda \Sigma}^{2}}{4 \pi}=0.048 \pm 0.005 \pm 0.004
$$

larger by about $25 \%$ than the value used in recent NSC one boson exchange models [32]. 


\section{III. $\Xi^{-}$ATOMS}

Some new information on the $\Xi^{-}$nucleus interaction has been recently reported from $\left(K^{-}, K^{+}\right)$counter experiments. Fukuda et al. [33] have shown fits to the very low energy part (including the bound state region) of the $\Xi^{-}$hypernuclear spectrum in the ${ }^{12} \mathrm{C}\left(K^{-}, K^{+}\right) X$ reaction on a scintillating fiber active target (KEK experiment E224), resulting in an estimate for the depth of the (attractive) $\Xi$ nucleus potential $\left(V_{0}^{(\Xi)}\right)$ between 15 to $20 \mathrm{MeV}$. The experimental energy resolution of about $10 \mathrm{MeV}$ in this experiment was too poor to allow identification of any bound state peak structure which could have given more definitive information on the well depth. A somewhat cleaner and better resolved spectrum has been recently presented by the Brookhaven AGS experiment E885 [34], suggesting that $V_{0}^{(\Xi)} \sim 14$ $\mathrm{MeV}$.

An earlier KEK experiment (E176) gave evidence for three events of stopped $\Xi^{-}$in light emulsion nuclei, each showing a decay into a pair of single $\Lambda$ hypernuclei. The first two events [35] are consistent energetically with a $\Xi^{-}$atomic state in ${ }^{12} \mathrm{C}$ bound by $B_{\Xi^{-}}\left({ }^{12} \mathrm{C}\right)=$ $0.58 \pm 0.14 \mathrm{MeV}$. However, this value could only be ascribed to capture from the $1 s$ state which is estimated to occur in less than $1 \%$ of the total number of captures. This binding energy is distinctly larger than the calculated value $B_{\Xi^{-}}^{2 p}\left({ }^{12} \mathrm{C}\right) \lesssim 0.32 \mathrm{MeV}$ for the $2 p$ state, for a wide range of strong-interaction potentials. Moreover, the $\Xi^{-}$capture probability in ${ }^{12} \mathrm{C}$ from $p$ states is a few percent at most. The most likely capture in ${ }^{12} \mathrm{C}$, as seen in Fig. [ taken from Ref. [36], occurs from the atomic 3d state. However, the binding of this atomic state $(126 \mathrm{keV})$ is essentially determined by the Coulomb potential, and its sensitivity to the $\Xi$-nucleus strong interaction assumed potential is of the order of 100 $\mathrm{eV}$, substantially smaller than a typical error of $100 \mathrm{keV}$ incurred in emulsion work. We point out that there exist alternative interpretations of these two events as captures on ${ }^{14} \mathrm{~N}$, with binding energies consistent with the calculated value of $175 \mathrm{keV}$, for example [35], $B_{\Xi^{-}}\left({ }^{14} \mathrm{~N}\right)=0.35 \pm 0.20 \mathrm{MeV}$. Furthermore, a likely interpretation of the third event 37 is due to capture on ${ }^{16} \mathrm{O}$, with $B_{\Xi^{-}}\left({ }^{16} \mathrm{O}\right)=0.31 \pm 0.23 \mathrm{MeV}$ (compared to the calculated value of $231 \mathrm{keV}$ ). Clearly, whereas these emulsion events are consistent with capture from $3 d$ atomic states, they are useless as a source of information regarding the $\Xi$-nucleus interaction.

An alternative source of information on the $\Xi$-nucleus strong interaction would be the measurement of X-ray energies from transitions between low lying levels of $\Xi^{-}$atoms, as suggested recently by Batty et al. [36]. The experimental accuracies of the proposed measurements are sufficient to obtain meaningful information on the $\Xi$-nucleus interaction. Full atomic cascade calculations have been performed [36] for $\Sigma^{-}$and $\Xi^{-}$atoms and confirmed, as expected, that the processes within these two hadronic atoms are very similar. The remaining major differences are in the production reactions. Whereas $\Sigma^{-}$hyperons are produced by the $p\left(K^{-}, \pi^{+}\right) \Sigma^{-}$reaction at rest, the $p\left(K^{-}, K^{+}\right) \Xi^{-}$reaction occurs at higher energies, thus causing decay losses during the slowing down time of the $\Xi^{-}$particle to be non-negligible. Prior to such an experiment it will be necessary to optimize the experimental setup, which includes a hydrogen production target, a heavy moderator such as $\mathrm{Pb}$ or $\mathrm{W}$, the target to be studied and the detectors, both for $\mathrm{X}$ rays and for the detection of the outgoing $K^{+}$, which is essential in order to reduce background. 


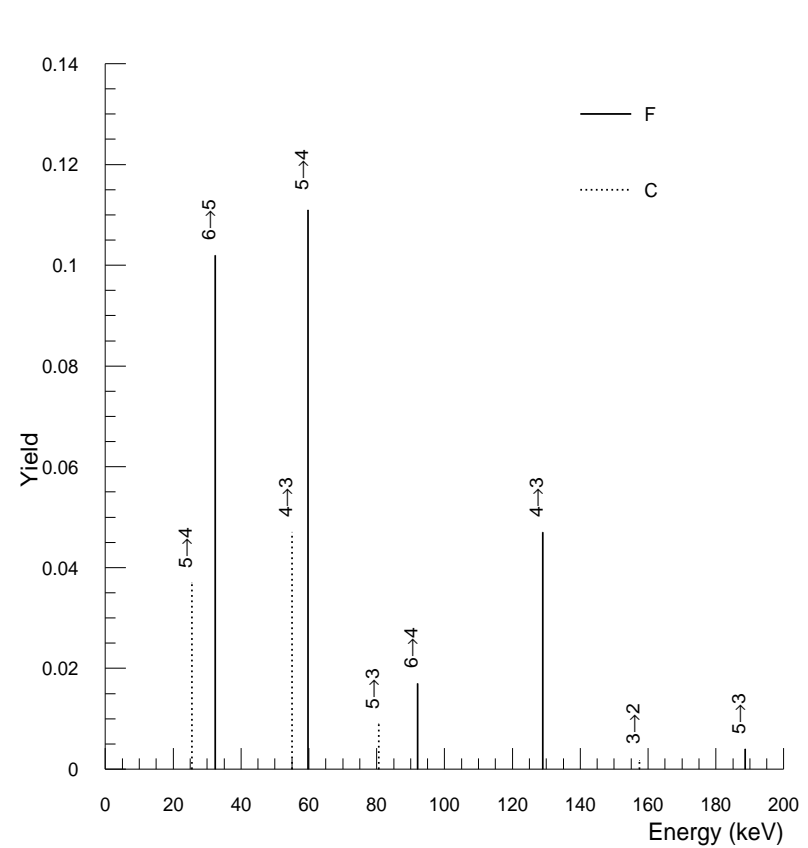

FIG. 7. Calculated $X i^{-}$X-ray spectrum for a teflon target.

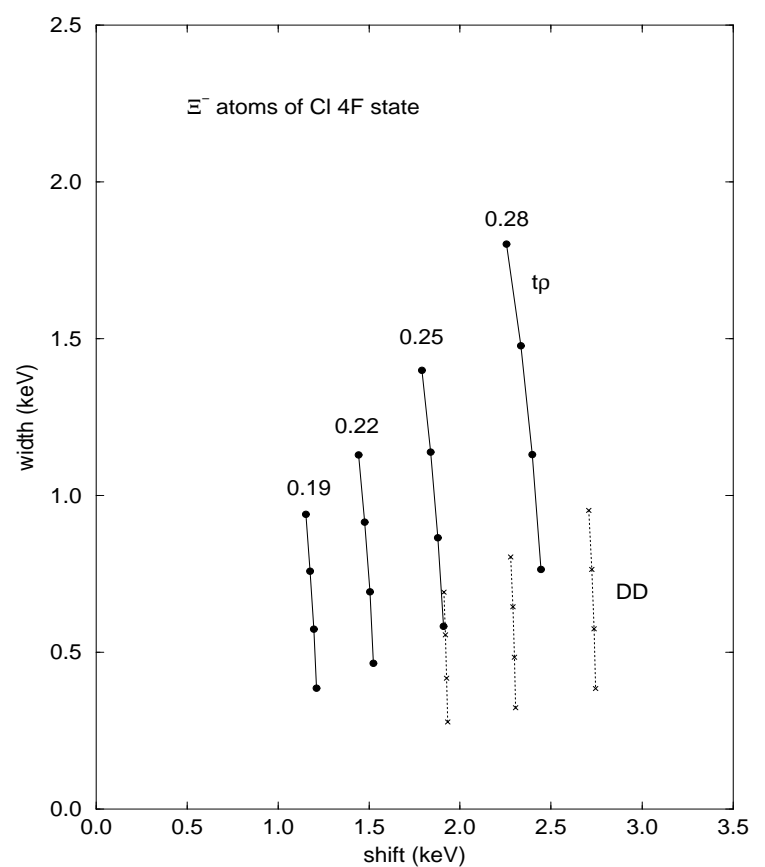

FIG. 8. Calculated shifts and widths for the $4 f X i^{-}$atomic state in $\mathrm{Cl}$ for different assumed optical potentials.

Adopting an attractive optical potential $V_{\text {opt }}$ for the $\Xi^{-}$nucleus strong interaction, and assuming a depth of 15-20 MeV with an imaginary part of 1-3 MeV, Batty et al. [36] were able to propose four targets suitable for X-ray measurements along the periodic table: $\mathrm{F}$, $\mathrm{Cl}$, I, Pb. A calculated X-ray spectrum for a teflon $\left(\mathrm{CF}_{2}\right)$ target is shown in Fig. 7 and it is seen that the presence of carbon in the target should not affect the possibility of observing transitions in $\Xi^{-} \mathrm{F}$ atoms. This choice of targets also proves to be a sensible one for moderate changes in $V_{\text {opt }}$. Nevertheless, even if the actual potential turns out to be very different from the one used in these calculations, only relatively small changes will result, because the whole phenomenon of hadronic atoms is dominated by the Coulomb interaction. Deriving strong-interaction level shifts and widths for as few as 2 to 4 measured X-ray spectra, it was shown by Friedman [10 that $V_{\text {opt }}$ may be constrained to a reasonable accuracy over a meaningful range of nuclear densities. The sensitivity to the $t \rho$ optical potential assumed in these calculations is demonstrated in Fig. 8 for $\mathrm{Cl}$. The solid curves connect shift and width values obtained for fixed values of Re $b_{0}$, listed above the lines. The four points along each line correspond to values of $\operatorname{Im} b_{0}$ from $0.05 \mathrm{fm}$ down to $0.02 \mathrm{fm}$. Similar results for a DD potential are also shown.

Extracting $V_{0}^{(\Xi)}$ directly from experiment would provide extremely valuable information on whether or not strange hadronic matter [38 exists. Indeed it was found 39] that once $V_{0}^{(\Xi)} \gtrsim 15 \mathrm{MeV}$, and for a strangeness fraction as small as about 0.1 in multi- $\Lambda$ hypernuclei, the free-space strong-interaction conversion $\Xi N \rightarrow \Lambda \Lambda$ becomes Pauli forbidden and the reverse process occurs. This means that $\Xi$ hyperons together with nucleons and $\Lambda$ hyperons may become stable against strong-interaction baryon emission, and multistrange nuclei with large strangeness fraction $-S / A \sim 1$ and small charge fraction $|Q| / A \ll 1 / 2$, decaying only weakly, should generallly exist; for a recent update see Ref. [40]. 


\section{ACKNOWLEDGEMENTS}

I would like to acknowledge a longstanding collaboration on the topics here reviewed with Drs. C.J. Batty, A. Cieply, E. Friedman and J. Mareš, as well as useful discussions with

Drs. A. Ramos and J. Schaffner-Bielich. This work was partly supported by the trilateral DFG contract GR 243/51-2.

[1] E. Friedman, A. Gal, J. Mareš and A. Cieply, Phys. Rev. C 60 (1999) 024314.

[2] J. Schaffner, A. Gal, I.N. Mishustin, H. Stoecker and W. Greiner, Phys. Lett. B 334 (1994) 268; J. Schaffner and I.N. Mishustin, Phys. Rev. C 53

[3] E. Friedman, A. Gal and C.J. Batty, Phys. Lett. B 308 (1993) 6.

[4] E. Friedman, A. Gal and C.J. Batty, Nucl. Phys. A 579 (1994) 518.

[5] C.J. Batty, E. Friedman and A. Gal, Phys. Rep. 287 (1997) 385.

[6] G.E. Brown and M. Rho, Nucl. Phys. A 596 (1996) 503.

[7] V. Koch, Phys. Lett. B 337 (1994) 7.

[8] T. Waas, N. Kaiser and W. Weise, Phys. Lett. B 365 (1996) 12; ibid. 379 (1996) 34.

[9] E. Oset and A. Ramos, Nucl. Phys. A 635 (1998) 99.

[10] E. Friedman, Nucl. Phys. A 639 (1998) 511c.

[11] M. Lutz, Phys. Lett. B 426 (1998) 12.

[12] A. Ramos and E. Oset, Nucl. Phys. A 671 (2000) 481.

[13] J. Schaffner-Bielich, V. Koch and M. Effenberger, Nucl. Phys. A 669 (2000) 153.

[14] S. Hirenzaki, Y. Okumura, H. Toki, E. Oset and A. Ramos, Phys. Rev. C 61 (2000) 055205.

[15] M. Mizoguchi, S. Hirenzaki, and H. Toki, Nucl. Phys. A 567 (1994) 893.

[16] A. Baca, C. Garcia-Recio and J. Nieves, Nucl. Phys. A 673 (2000) 335.

[17] A. Cieply, E. Friedman, A. Gal and J. Mareš, in preparation (2001).

[18] E. Friedman and G. Soff, J. Phys. G: Nucl. Phys. 11 (1985) L37.

[19] H. Toki and T. Yamazaki, Phys. Lett. B 213 (1988) 129.

[20] T. Yamazaki et al., Z. Phys. A 355 (1996) 219; H. Gilg et al., Phys. Rev. C 62 (2000) 025201;

K. Itahashi et al., ibid. 025202.

[21] E. Friedman and A. Gal, Phys. Lett. B 459 (1999) 43.

[22] E. Friedman and A. Gal, Nucl. Phys. A 658 (1999) 345.

[23] A. Gal, E. Friedman and C.J. Batty, Phys. Lett. B 491 (2000) 219.

[24] C.J. Batty, E. Friedman and A. Gal, Nucl. Phys. A (in press).

[25] J. Nieves and E. Oset, Nucl. Phys. A 518 (1990) 617.

[26] T. Kishimoto, Phys. Rev. Lett. 83 (1999) 4701.

[27] Y. Akaishi and T. Yamazaki, in Physics and Detectors for DA $\Phi$ NE, eds. S. Bianco et al., Frascati Physics Series Vol. 16 (1999) 59.

[28] C.J. Batty, E. Friedman and A. Gal, Phys. Lett. B 335 (1994) 273; Prog. Theor. Phys. Suppl. 117 (1994) 227.

[29] J. Mareš, E. Friedman, A. Gal and B.K. Jennings, Nucl. Phys. A 594 (1995) 311.

[30] J. Dabrowski, Phys. Rev. C 60 (1999) 025205.

[31] B. Loiseau and S. Wycech, Phys. Rev. C 63 (2001) (in press). 
[32] V.G.J. Stoks and Th.A. Rijken, Phys. Rev. C 59 (1999) 3009.

[33] T. Fukuda et al., Phys. Rev. C 58 (1998) 1306.

[34] P. Khaustov et al., Phys. Rev. C 61 (2000) 054603.

[35] S. Aoki et al., Prog. Theor. Phys. 89 (1993) 493; Phys. Lett. B 355 (1995) 45.

[36] C.J. Batty, E. Friedman and A. Gal, Phys. Rev. C 59 (1999) 295.

[37] K. Nakazawa, T. Sasaki and Y. Yamamoto, in Proc. Workshop Hyp. Physics, eds. T. Motoba, Y. Akaishi and T. Nagae [Genshikaku Kenkyu 41(6) (1997) 75].

[38] J. Schaffner, C.B. Dover, A. Gal, C. Greiner and H. Stöcker, Phys. Rev. Lett. 71 (1993) 1328; J. Schaffner, C.B. Dover, A. Gal, C. Greiner, D.J. Millener and H. Stöcker, Ann. Phys. [NY] 235 (1994) 35.

[39] S. Balberg, A. Gal and J. Schaffner, Prog. Theor. Phys. Suppl. 117 (1994) 325.

[40] J. Schaffner-Bielich and A. Gal, Phys. Rev. C 62 (2000) 034311. 